\title{
Utilidad de un sistema de seguimiento óptico de instrumental en cirugía laparoscópica para evaluación de destrezas motoras
}

\author{
Juan A. Sánchez-Margallo , Francisco M. Sánchez-Margallo , José B. Pagador Carrasco \\ Ignacio Oropesa García , Enrique J. Gómez Aguilera - y José Moreno del Pozo
}

R E S U M E N

Introducción: En este trabajo se estudia la utilidad de un sistema de evaluación de destrezas quirúrgicas basado en el análisis de los movimientos del instrumental laparoscópico.

Método: El sistema consta de un simulador físico laparoscópico y un sistema de seguimiento y evaluación de habilidades técnicas quirúrgicas. En el estudio han participado 6 cirujanos con experiencia intermedia (entre 1 y 50 intervenciones laparoscópicas) y 5 cirujanos expertos (más de 50 intervenciones laparoscópicas), todos ellos con la mano derecha como dominante. Cada sujeto realizó 3 repeticiones de una tarea de corte con la mano derecha en tejido sintético, una disección de la serosa gástrica y una sutura en la disección realizada. Para cada ejercicio se analizaron los parámetros de tiempo, distancia recorrida, velocidad, aceleración y suavidad de movimientos para los instrumentos de ambas manos.

Resultados: En la tarea de corte, los cirujanos expertos muestran menor aceleración ( $\mathrm{p}=0,014$ ) y mayor suavidad en los movimientos $(\mathrm{p}=0,023)$ en el uso de la tijera. Respecto a la actividad de disección, los cirujanos expertos requieren menos tiempo $(p=0,006)$ y recorren menos distancia con ambos instrumentos ( $p=0,006$ para disector y $p=0,01$ para tijera). En la tarea de sutura, los cirujanos expertos presentan menor tiempo de ejecución que los cirujanos de nivel intermedio $(p=0,037)$ y recorren menos distancia con el disector $(p=0,041)$.

Conclusiones: El sistema de evaluación se mostró útil en las tareas de corte, disección y sutura, y constituye un progreso en el desarrollo de sistemas avanzados de entrenamiento y evaluación de destrezas quirúrgicas laparoscópicas. 


\title{
Usefulness of an optical tracking system in laparoscopic surgery for motor skills assessment
}

\begin{abstract}
A B S T R A C T
Introduction: The objective of this study is to assess the usefulness of an evaluation system of surgical skills based on motion analysis of laparoscopic instruments.

Method: This system consists of a physical laparoscopic simulator and a tracking and assessment system of technical skills in laparoscopy. Six surgeons with intermediate experience (between 1 and 50 laparoscopic surgeries) and 5 experienced surgeons (more than 50 laparoscopic surgeries) took part in this study. All participants were right-handed. The subjects performed 3 repetitions of a cutting task on synthetic tissue with the right hand, dissection of a gastric serous layer, and a suturing task in the dissection previously done. Objective metrics such as time, path length, speed of movements, acceleration and motion smoothness were analyzed for the instruments of each hand.

Results: In the cutting task, experienced surgeons show less acceleration ( $P=.014)$ and a smoother motion $(P=.023)$ using the scissors. Regarding the dissection activity, experienced surgeons need less time $(P=.006)$ and less length with both instruments $(P=.006$ for dissector and $P=.01$ for scissors). In the suturing task, experienced surgeons require less time $(\mathrm{P}=.037)$ and distance travelled $(\mathrm{P}=.041)$ by the dissector.

Conclusions: This study shows the usefulness of the evaluation system for the cutting, dissecting, and suturing tasks. It represents a significant step in the development of advanced systems for training and assessment of surgical skills in laparoscopic surgery.
\end{abstract}

\section{Introducción}

La cirugía laparoscópica es una disciplina quirúrgica con elevados requerimientos técnicos para el cirujano, como el uso de nuevo instrumental ${ }^{1}$, la carencia de sensación táctil, el efecto de movimiento inverso (conocido como «efecto fulcrum») o la pérdida de sensación de profundidad. Este hecho supone un importante reto para el cirujano, en cuanto a la adquisición de nuevas habilidades psicomotoras, para estar capacitado en la realización de intervenciones quirúrgicas seguras ${ }^{2,3}$.

Sería conveniente conocer las habilidades psicomotoras de los cirujanos en fase de formación como una parte esencial en la evaluación de su competencia quirúrgica. Por esta razón, autores, cirujanos y asociaciones piden cada vez más el desarrollo de herramientas de evaluación que acrediten a los cirujanos como técnicamente competentes ${ }^{3-5}$.

Para cubrir esta necesidad, se han desarrollado diversos sistemas de formación y evaluación quirúrgica que evitan poner en riesgo al paciente, favorecen su uso fuera del entorno clínico, así como la objetividad y la automatización de los procesos de evaluación de los cirujanos. Principalmente, estos sistemas se clasifican en 2 grandes grupos: sistemas virtuales y físicos ${ }^{6}$. En este trabajo nos centraremos en los sistemas físicos de formación y evaluación quirúrgica por su mayor realismo en el uso del instrumental, así como en la interacción con el entorno de trabajo ${ }^{7,8}$. Actualmente, se dispone de sistemas con diversas características y aplicaciones tanto comerciales como académicos. Sin embargo, no existe un sistema universalmente extendido o recomendado como herramienta objetiva de evaluación de las habilidades quirúrgicas ${ }^{3}$.

El análisis de los movimientos del instrumental es un método efectivo para la evaluación objetiva de las habilidades psicomotoras del cirujano $0^{6,9,10}$. Durante el aprendizaje de una tarea, el sujeto ejecuta movimientos más eficientes cuando llega a dominar esta tarea ${ }^{11}$. Para registrar esta información se han utilizado diversas tecnologías (electromagnéticas, mecánicas, ópticas) con las que llevar a cabo el seguimiento del instrumental durante la actividad de evaluación ${ }^{12-14}$.

Diferentes autores han analizado diversos parámetros basados en el movimiento del instrumental laparoscópico para llevar a cabo la evaluación objetiva de las habilidades técnicas del cirujano ${ }^{6,9}$. Estos parámetros pueden predecir el nivel de experiencia del cirujano de forma tan precisa como con el uso de escalas de certificación ${ }^{15}$. Para hacer uso de estas medidas de evaluación, con el fin de diferenciar entre cirujanos con distintos niveles de experiencia, es necesario establecer cuáles de ellas son determinantes para cada tarea a evaluar.

El objetivo principal de este estudio es analizar la utilidad de un sistema de evaluación de las destrezas psicomotoras en cirugía laparoscópica, basado en el análisis del movimiento de los instrumentos laparoscópicos. Este estudio se ha llevado a cabo en 2 grupos de cirujanos de diferente experiencia en cirugía laparoscópica durante un conjunto de tareas básicas de formación en simulador, como son el corte en tejido sintético, la disección de la serosa gástrica y la sutura en tejido orgánico.

\section{Método}

\section{El simulador}

El sistema se compone de 2 módulos: un simulador físico para el entrenamiento de habilidades laparoscópicas (SIMULAP ${ }^{\circledR}$, CCMIJU, Cáceres, España) $)^{7,16}$ y un sistema de seguimiento y 


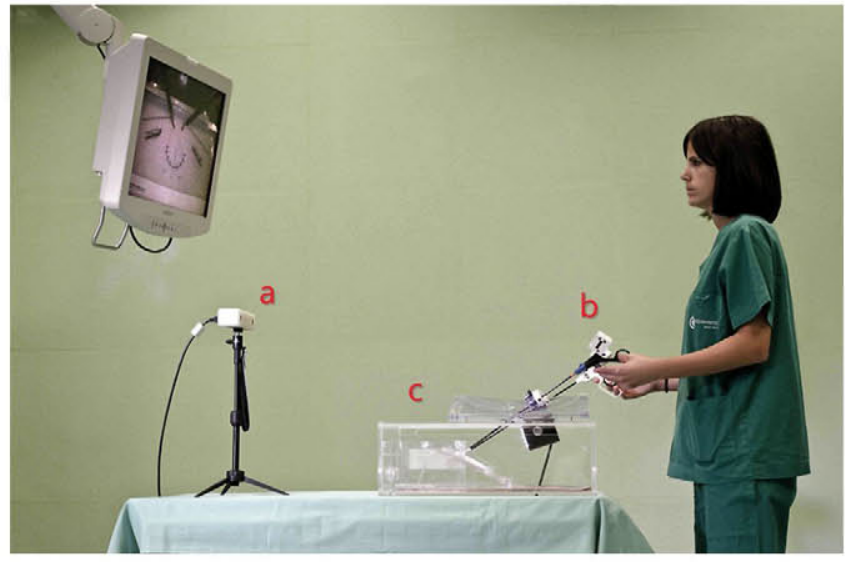

Figura 1 - Sistema de formación y evaluación de destrezas quirúrgicas: a) Sistema de seguimiento. b) Instrumental laparoscópico con marcas artificiales de seguimiento. c) Simulador de entrenamiento.

evaluación de las habilidades técnicas quirúrgicas (fig. 1). Para las tareas de evaluación se han utilizado: una tijera laparoscópica (Endo Shears, Auto Suture, Covidien, Mansfield, MA, EE. UU.), un disector laparoscópico (Richard Wolf $\mathrm{GmbH}$, Knittlingen, Alemania) y un portaagujas laparoscópico (Karl Storz GmbH \& Co. KG, Tuttlingen, Alemania).

\section{Sistema de seguimiento del instrumental laparoscópico}

Este sistema consta de un dispositivo óptico de tercera generación (MicronTracker ${ }^{\circledR}$, 3Hx60, Claron Technology Inc., Toronto, Canadá), el cual ha sido adaptado para su utilización con instrumental laparoscópico real. El sistema utiliza marcas artificiales identificables en el espectro visible. Para cada instrumental empleado se ha diseñado un soporte con 3 marcas artificiales que ofrecen una visión frontal y lateral de cada instrumental, de modo que mejoren su rango de localización (fig. 2). El soporte es de un diseño ligero y robusto, y no interfiere en el uso habitual del instrumental. Previamente al análisis de la información, se ha aplicado un filtro Kalman de suavizado $^{17}$ a la señal registrada de cada instrumento.

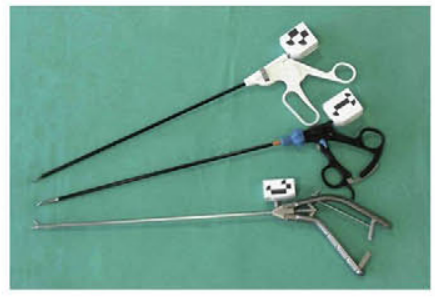

Figura 2 - Detalle del diseño y localización de las marcas artificiales en cada tipo de instrumental.

\section{Sujetos}

En el estudio han participado 6 cirujanos con experiencia intermedia (entre una y 50 intervenciones laparoscópicas) y 5 cirujanos expertos (con más de 50 intervenciones laparoscópicas), todos ellos con la mano derecha como dominante. Al no existir un criterio estándar para la definición de la pericia quirúrgica, se ha tomado como valor de clasificación el número de intervenciones quirúrgicas realizadas. Concretamente, el grupo de cirujanos intermedios ha realizado un promedio de 17,6 intervenciones laparoscópicas (en un rango de entre 7 y 25 intervenciones) y los cirujanos expertos un promedio de 85 intervenciones (en un rango de entre 50 y más de 150 intervenciones). Al inicio de la actividad todos los participantes rellenaron un cuestionario sobre información demográfica y experiencia quirúrgica.

\section{Tareas y parámetros de evaluación}

Cada sujeto realizó 3 tareas de formación básica laparoscópica, con diferente grado de complejidad y sin tiempo límite de finalización. En primer lugar, se llevó a cabo una tarea de corte en una pletina de tejido sintético, siguiendo un patrón establecido y utilizando la mano dominante. Seguidamente, se efectuó una disección de la capa serosa de un estómago de cadáver porcino a lo largo de una incisión de $3 \mathrm{~cm}$, procurando su separación de las capas musculares. Finalmente, se llevó a cabo una sutura en la disección realizada. La tarea de sutura se realizó en 4 fases: paso de la aguja, un anudado doble y 2 anudados simples en sentidos opuestos. Todas las condiciones del estudio fueron estandarizadas para todos los sujetos. Para el ejercicio de corte y disección, se utilizó un disector en la

\section{Tabla 1 - Descripción de los parámetros de evaluación analizados}

\begin{tabular}{lll} 
Métrica & \multicolumn{1}{c}{ Definición } & Valor \\
\hline Tiempo $(\mathrm{s})$ & Tiempo en realizar la tarea & $T=t_{f}-t_{0}$ \\
Distancia recorrida $(\mathrm{mm})$ & $\begin{array}{l}\text { Longitud de la trayectoria descrita por la punta del } \\
\text { instrumental durante la realización de la tarea }\end{array}$ & $\int_{t-0}^{T} \sqrt{\left(\frac{d r_{t}(t)}{d t}\right)^{2} d t}$ \\
Velocidad $(\mathrm{mm} / \mathrm{s})$ & $\begin{array}{l}\text { Índice de la variación de la posición del instrumental } \\
\text { con respecto al tiempo durante el transcurso de la tarea }\end{array}$ & $\frac{1}{T} \int_{t-0}^{T} \sqrt{\left(\frac{d r_{x}}{d t}\right)^{2}+\left(\frac{d r_{y}}{d t}\right)^{2}+\left(\frac{d r_{z}}{d t}\right)^{2}}$ \\
Aceleración $\left(\mathrm{mm} / \mathrm{s}^{2}\right)$ & $\begin{array}{l}\text { Indice de la variación de la velocidad del instrumental } \\
\text { con respecto al tiempo durante el transcurso de la tarea }\end{array}$ & $\frac{1}{T} \int_{t-0}^{T} \sqrt{\left(\frac{d^{2} r_{x}}{d t^{2}}\right)^{2}+\left(\frac{d^{2} r_{y}}{d t^{2}}\right)^{2}+\left(\frac{d^{2} r_{z}}{d t^{2}}\right)^{2}}$ \\
Suavidad de movimientos $\left(\mathrm{mm} / \mathrm{s}^{3}\right)$ & $\begin{array}{l}\text { Cambios en la aceleración del instrumental que } \\
\text { provocan movimientos bruscos durante la tarea }\end{array}$ & $\frac{1}{T} \int_{t-0}^{T} \sqrt{\left(\frac{d^{3} r_{x}}{d t^{3}}\right)^{2}+\left(\frac{d^{3} r_{y}}{d t^{3}}\right)^{2}+\left(\frac{d^{3} r_{z}}{d t^{3}}\right)^{2}}$
\end{tabular}


Tabla 2 - Comparativa de los parámetros de evaluación de las destrezas motoras durante la tarea de corte en tejido sintético

\begin{tabular}{|c|c|c|c|c|c|}
\hline \multirow[t]{2}{*}{ Variable } & \multicolumn{2}{|c|}{ Experiencia intermedia } & \multicolumn{2}{|c|}{ Expertos } & \multirow[t]{2}{*}{$\mathrm{p}$} \\
\hline & Valor medio & IC & Valor medio & IC & \\
\hline Tiempo & 100,7 & $73,5-127,9$ & 90,0 & $80,5-99,6$ & 0,56 \\
\hline Distancia recorrida $\mathrm{MD}$ & $6.882,1$ & $4.167,9-9.596,5$ & $5.310,4$ & $4.787,6-5.833,2$ & 0,298 \\
\hline Distancia recorrida MI & $2.826,1$ & $2.508,8-3.143,3$ & $2.308,8$ & $1.911,2-2.706,4$ & 0,145 \\
\hline Velocidad máx. MD & 590,4 & $352,9-827,9$ & 555,4 & $381,0-729,9$ & 0,783 \\
\hline Velocidad máx. MI & 234,2 & $192,3-276,0$ & 217,8 & $141,5-294,2$ & 0,057 \\
\hline Velocidad media MD & 67,4 & $59,6-75,3$ & 60,2 & $56,0-64,5$ & 0,314 \\
\hline Velocidad media MI & 29,5 & $24,0-35,0$ & 26,3 & $21,9-30,8$ & 0,408 \\
\hline Aceleración máx. MD & $4.310,7$ & $2.689,6-5.931,9$ & $3.321,9$ & $2.526,5-4.117,2$ & 0,121 \\
\hline Aceleración máx. MI & $2.108,6$ & $1.723,2-2.494,0$ & $1.841,2$ & $1.405,4-2.277,1$ & 0,22 \\
\hline Aceleración media MD & 438,1 & $397,0-479,3$ & 367,6 & $354,5-380,8$ & $0,014^{\circ}$ \\
\hline Aceleración media MI & 208,6 & $163,1-254,2$ & 174,1 & $146,2-202,1$ & 0,232 \\
\hline Suavidad de movimientos máx. MD & $29.037,4$ & $18.424,2-39.650,6$ & $27.701,8$ & $22.369,5-33.034,1$ & 0,211 \\
\hline Suavidad de movimientos máx. MI & $13.632,7$ & $11.315,8-15.949,6$ & $12.761,5$ & $9.739,8-15.783,2$ & 0,44 \\
\hline Suavidad de movimientos media $\mathrm{MD}$ & $2.285,5$ & $2.072,7-2.498,4$ & $1.913,5$ & $1.829,9-1.997,1$ & $0,023^{\circ}$ \\
\hline Suavidad de movimientos media MI & $1.191,4$ & $950,2-1.432,6$ & $1.016,3$ & $855,7-1.176,9$ & 0,121 \\
\hline
\end{tabular}

mano izquierda y una tijera en la derecha. En el ejercicio de sutura, un disector en la mano izquierda y un portaagujas en la derecha. Los sujetos siguieron la tarea por medio de un monitor que les mostraba el vídeo procedente de la cámara del interior del simulador.

Para la evaluación de las habilidades técnicas de cada sujeto, se han calculado una serie de métricas objetivas ${ }^{6,18}$ basadas en el uso de los instrumentos laparoscópicos durante cada tarea (tabla 1). Se realizaron 3 repeticiones de cada tarea, de las cuales se obtuvo el valor medio de cada parámetro de evaluación, para el instrumento de la mano izquierda y de la derecha.

\section{Análisis estadístico}

El estudio comparativo entre parámetros de evaluación se realizó entre los 2 grupos de cirujanos y para cada tarea. El análisis estadístico se llevó a cabo mediante el software SPSS (versión 15.0, SPSS Inc., Chicago, IL, EE. UU.) y se utilizó la prueba $U$ de Mann-Whitney para comparar las diferencias estadísticas entre ambos grupos, usando un valor de significación de $\mathrm{p}<0,05$.

\section{Resultados}

Durante la tarea de corte en tejido sintético, se han obtenido diferencias significativas en los parámetros de aceleración media y suavidad media de movimientos en el uso de la tijera laparoscópica (tabla 2).

Respecto al ejercicio de disección, los valores de tiempo y distancia recorrida por ambos instrumentos son claramente menores para el grupo de cirujanos expertos (tabla 3). Sin

Tabla 3 - Comparativa de los parámetros de evaluación de las destrezas motoras durante la tarea de disección en tejido orgánico

\begin{tabular}{|c|c|c|c|c|c|}
\hline \multirow[t]{2}{*}{ Variable } & \multicolumn{2}{|c|}{ Experiencia intermedia } & \multicolumn{2}{|c|}{ Experto } & \multirow[t]{2}{*}{$\mathrm{p}$} \\
\hline & Valor medio & IC & Valor medio & IC & \\
\hline Tiempo & 179,5 & $144,5-214,6$ & 76,2 & $59,4-93,0$ & $0,006^{\circ}$ \\
\hline Distancia recorrida $\mathrm{MD}$ & $8.185,6$ & $6.872,7-9.498,5$ & $3.712,0$ & $2.767,3-4.656,7$ & $0,006^{\circ}$ \\
\hline Distancia recorrida MI & $6.628,8$ & $5.253,2-8.004,5$ & $3.120,7$ & $2.088,5-4.152,8$ & $0,01^{*}$ \\
\hline Velocidad máx. MD & 502,4 & $409,7-595,2$ & 382,6 & $218,8-546,3$ & 0,308 \\
\hline Velocidad máx. MI & 281,0 & $227,6-334,5$ & 311,8 & $220,3-403,2$ & 0,497 \\
\hline Velocidad media MD & 46,7 & $44,0-49,4$ & 48,9 & $45,7-52,2$ & 0,396 \\
\hline Velocidad media MI & 37,4 & $35,7-39,1$ & 41,4 & $33,3-49,4$ & 0,174 \\
\hline Aceleración máx. MD & $3.165,9$ & $2.691,9-3.639,8$ & $2.685,2$ & $2.252,7-3.117,7$ & 0,308 \\
\hline Aceleración máx. MI & $2.248,9$ & $1.994,2-2.503,7$ & $2.171,9$ & $2.012,9-2.331,0$ & 0,734 \\
\hline Aceleración media MD & 294,3 & $281,8-306,8$ & 313,7 & $272,2-355,3$ & 0,168 \\
\hline Aceleración media MI & 248,1 & $234,0-262,3$ & 285,3 & $217,3-353,4$ & 0,153 \\
\hline Suavidad de movimientos máx. MD & $18.249,3$ & $14.982,0-21.516,6$ & $18.302,3$ & $14.670,8-21.933,9$ & 0,865 \\
\hline Suavidad de movimientos máx. MI & $15.400,7$ & $13.249,2-17.552,2$ & $14.319,9$ & $13.484,8-15.155,1$ & 0,497 \\
\hline Suavidad de movimientos media MD & $1.558,8$ & $1.497,6-1.620,0$ & $1.678,2$ & $1.475,9-1.880,5$ & 0,234 \\
\hline Suavidad de movimientos media MI & $1.341,7$ & $1.258,7-1.424,8$ & $1.571,0$ & $1.192,5-1.949,4$ & 0,182 \\
\hline
\end{tabular}

IC: intervalo de confianza al 95\%; MD: mano derecha; MI: mano izquierda.

${ }^{*} \mathrm{p}<0,05$ 
Tabla 4 - Comparativa de los parámetros de evaluación de las destrezas motoras durante la tarea de sutura en tejido orgánico

Variable

Experiencia intermedia

Valor medio

IC

Experto

Tiempo

204,5

Distancia recorrida $\mathrm{MD}$

$7.465,7$

$8.457,7$

Distancia recorrida MI

535,3

Velocidad máx. MI

Velocidad media MD

Velocidad media MI

Aceleración máx. MD

Aceleración máx. MI

Aceleración Media MD

306,9

38,8

41,6

$3.144,2$

$2.577,0$

244,7

281,8

Aceleración media MI

$18.693,2$

$17.479,4$

Suavidad de movimientos máx. MI

Suavidad de movimientos media MD

$1.306,8$

$1.515,7$

$\begin{array}{cr}162,9-246,0 & 135,8 \\ 5.833,4-9.098,0 & 6.304,0 \\ 6.556,7-10.358,6 & 5.303,4 \\ 435,0-635,6 & 368,4 \\ 238,6-375,2 & 299,4 \\ 33,9-43,8 & 45,7 \\ 38,4-44,8 & 41,8 \\ 2.602,7-3.685,6 & 2.594,4 \\ 2.185,2-2.968,7 & 2.488,7 \\ 206,2-283,2 & 289,7 \\ 255,7-307,9 & 277,2 \\ 15.997,3-21.389,2 & 14.473,6 \\ 14.291,6-20.667,3 & 15.715,7 \\ 1.100,7-1.512,8 & 1.525,9 \\ 1.390,5-1.640,8 & 1.483,2\end{array}$

Valor medio

135,8

$6.304,0$

$5.303,4$

368,4

299,4

45,7

$2.594,4$

$2.488,7$

289,7

277,2

$15.715,7$

$1.525,9$

Suavidad de movimientos media MI

$1.390,5-1.640,8$

IC: intervalo de confianza al 95\%; MD: mano derecha; MI: mano izquierda.

${ }^{*} \mathrm{p}<0,05$.

embargo, cabe destacar que en el caso del ejercicio de sutura en tejido orgánico, aparte del tiempo de ejecución del ejercicio, únicamente la distancia recorrida por el disector muestra diferencias significativas entre ambos grupos, siendo ambos parámetros menores en el caso del grupo experto (tabla 4).

Teniendo en cuenta las curvas de aprendizaje para el ejercicio de sutura (fig. 3), los cirujanos de nivel intermedio muestran un decremento importante en el tiempo de ejecución de la tarea y de la distancia recorrida por ambos instrumentos. Sin embargo, presentan un aumento en la velocidad y aceleración máxima en el uso del portaagujas, así como de la suavidad de movimientos máxima para ambos tipos de instrumental quirúrgico. Los cirujanos expertos reflejan un decremento en la aceleración y suavidad de movimientos máxima en el uso del portaagujas. En ambos grupos, aumenta la aceleración y suavidad de movimientos media en el uso del disector.

\section{Discusión}

La creciente necesidad de elaborar nuevos métodos de evaluación objetiva de las destrezas quirúrgicas en laparoscopia ha llevado al desarrollo de diversos sistemas de evaluación ${ }^{19,20}$. Sin embargo, esta tarea requiere de procedimientos más automáticos, como los basados en el análisis de los movimientos del instrumental. Para el desarrollo de este tipo de sistemas, bien sea para su aplicación en cirugía guiada por imagen ${ }^{21}$, para cirugía robótica ${ }^{22}$ o bien para evaluación de destrezas, se han utilizado diversas tecnologías de seguimiento como sistemas electromagnéticos ${ }^{13}$, mecánicos ${ }^{23}$, ópticos ${ }^{24}$ o basados en vídeo ${ }^{12,14}$.

En este artículo se propone un diseño novedoso para aplicar un sistema de seguimiento óptico de tercera generación para su uso durante la formación y la evaluación objetiva de las habilidades psicomotoras en cirugía laparoscópica. Se ha analizado la utilidad del sistema en 3 ejercicios de formación básica. De este modo, podemos determinar si el sistema es capaz de diferenciar entre cirujanos con experiencia intermedia y expertos. Los resultados obtenidos en estudios previos, respecto a la precisión del sistema de seguimiento utilizado, reflejan valores aptos para su uso como herramienta de análisis de los movimientos del instrumental, aunque se requiere una mejora de esta precisión para su aplicación en sistemas de cirugía guiada por imagen ${ }^{25}$. El sistema presentado ofrece una solución original en cuanto a la evaluación objetiva y automática de las destrezas quirúrgicas, sin interferir en la ergonomía del instrumental.

Respecto a los resultados obtenidos en este estudio, ningún parámetro de evaluación ha mostrado diferencias estadísticamente significativas en las 3 tareas evaluadas. Esto nos lleva a pensar que no es posible determinar de forma precisa el nivel de pericia del cirujano con una única medida de evaluación para las 3 tareas evaluadas ${ }^{10}$. Chmarra et al., en estudios previos, también establecieron que sería recomendable llevar a cabo la evaluación de las habilidades psicomotoras del cirujano con, al menos, 2 parámetros de evaluación ${ }^{8}$.

Se ha demostrado la eficacia del sistema en la evaluación de la tarea de corte respecto a las métricas de aceleración y suavidad de movimientos medias en el uso de la tijera laparoscópica. Para el mismo ejercicio, Pellen et al., aparte de la suavidad de movimientos, obtuvieron diferencias significativas respecto al tiempo y la distancia media recorrida por ambos instrumentos ${ }^{26}$. Respecto al presente estudio, consideramos que factores como la experiencia previa en tareas básicas laparoscópicas de ambos grupos de cirujanos y la sencillez de la tarea analizada conllevan que no se presenten más parámetros diferenciadores entre grupos.

Durante la tarea de disección, los cirujanos expertos requieren menos de la mitad del tiempo empleado por los cirujanos con experiencia intermedia para completar la tarea. Además, el grupo de cirujanos expertos muestra una velocidad media de uso de ambos instrumentos más elevada. Sin embargo, este grupo también muestran movimientos expeditivos durante el uso de ambos tipos de instrumental. Esto puede deberse a que la experiencia conlleva movimientos más 
Curva de aprendizaje - Tiempo Ejercicio de sutura

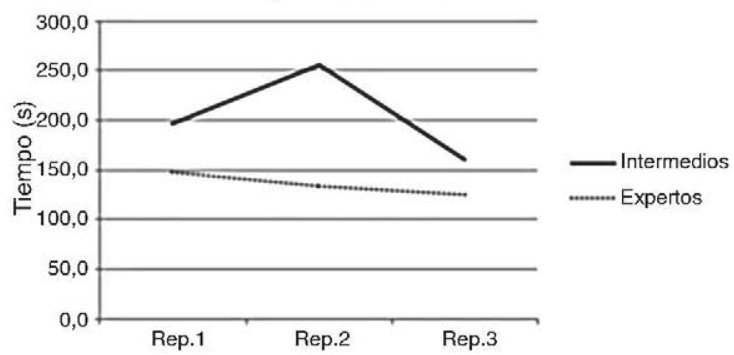

Curva de aprendizaje - Velocidad máxima Ejercicio de sutura

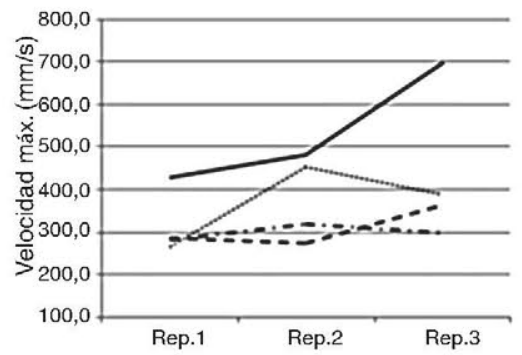

Curva de aprendizaje - Aceleración máxima Ejercicio de sutura

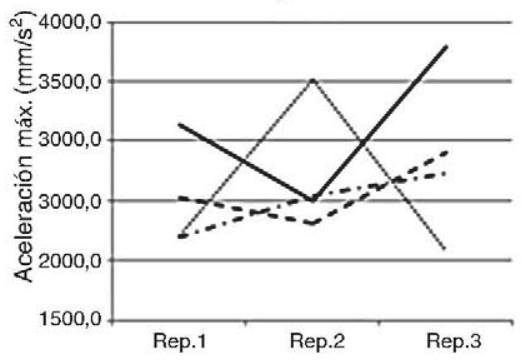

—Intermedios MD - - - Intermedios MI ......... Expertos MD -.- Expertos MI

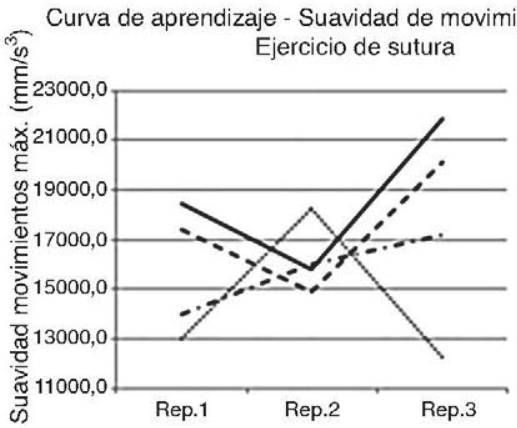

- Intermedios MD - - Intermedios MI -no...- Expertos MD -.- Expertos MI
Curva de aprendizaje - Distancia recorrida Ejercicio de sutura

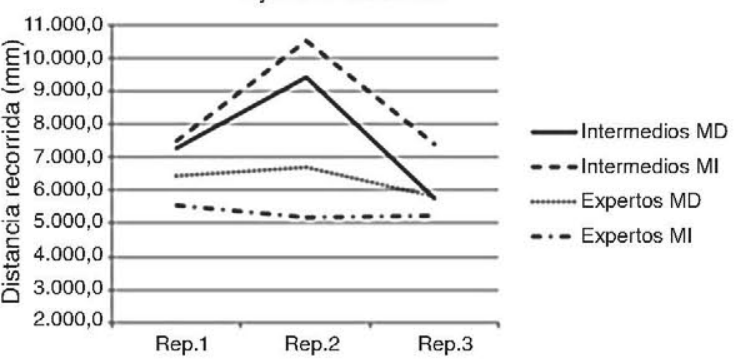

Curva de aprendizaje - Velocidad media Ejercicio de sutura

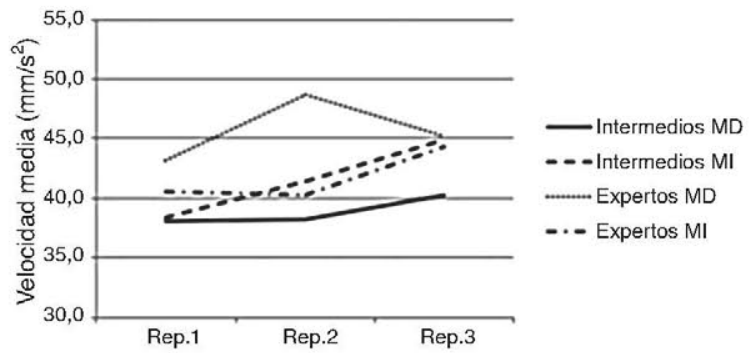

Curva de aprendizaje - Aceleración media Ejercicio de sutura

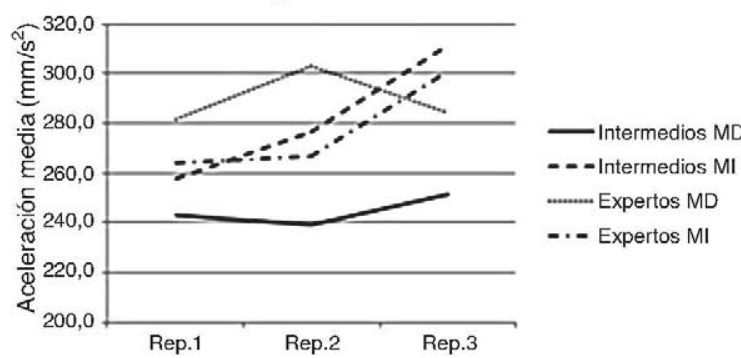

Curva de aprendizaje - Suavidad de movimientos media

कू

Figura 3 - Curvas de aprendizaje para las métricas motoras analizadas durante la tarea de sutura. Resultados del grupo de cirujanos de nivel intermedio y expertos respecto al instrumental de la mano derecha (MD) e izquierda (MI).

rápidos y, en ocasiones, con menor delicadeza, frente a la posible inseguridad presente en determinados momentos en el grupo de cirujanos de nivel intermedio.

Para la tarea de sutura se han obtenido diferencias estadísticamente significativas en los parámetros de tiempo y distancia recorrida por el disector. Estos resultados coinciden con los obtenidos en el trabajo de Yamaguchi et al. ${ }^{27}$ para cirujanos de diversos niveles de experiencia. Van Sickle et al. ${ }^{28}$ analizaron el paso de la aguja utilizando un patrón de látex, tarea en la que obtuvieron diferencias significativas en el tiempo de ejecución, distancia recorrida media y suavidad de movimiento media de ambos instrumentos para grupos de cirujanos noveles y expertos.

En otros trabajos, la tarea de sutura ha sido evaluada mediante un dispositivo electromagnético adherido a la palma de la mano del cirujano, del cual se ha demostrado su utilidad en la evaluación de destrezas con respecto a las métricas de tiempo de ejecución y número de movimientos ${ }^{29,30}$. En 
estudios previos ${ }^{13}$ hemos utilizado el mismo dispositivo pero, en este caso, para analizar el movimiento del instrumental durante una tarea de sutura laparoscópica, realizada por cirujanos noveles, de experiencia intermedia y expertos. La tarea de sutura fue dividida en 4 subtareas, tal y como hemos contemplado en el presente estudio, analizadas de forma individual. Los resultados respecto al grupo de cirujanos con experiencia intermedia y expertos mostraron diferencias significativas en la distancia recorrida por el portaagujas durante la subtarea de anudado doble. Sin embargo, no se obtuvieron diferencias significativas respecto el uso del disector, como se describe en los resultados obtenidos en los trabajos de Yamaguchi et al. ${ }^{27}$, van Sickle et al. ${ }^{28}$ o en el presente estudio. Por consiguiente, esto nos lleva a pensar que, además del tiempo de ejecución de la tarea, la distancia recorrida por los instrumentos es un factor presente en la mayoría de los estudios que analizan la sutura laparoscópica y que sería conveniente ampliar la muestra de estudio para poder establecer de forma más precisa qué instrumental (disector, portaagujas o ambos) es determinante en la diferenciación del nivel de habilidad técnica entre cirujanos para esta tarea.

Si analizamos la evolución de ambos grupos durante la tarea de sutura, apreciamos una clara mejora de los cirujanos de experiencia intermedia respecto al tiempo de ejecución y la distancia recorrida por ambos instrumentos. Los movimientos de los cirujanos expertos son más rápidos para el uso del portaagujas: esto parece estar claramente relacionado con el nivel de destreza para esta tarea. Sin embargo, ambos grupos presentan una velocidad de movimiento similar para el uso del disector. Por otro lado, los 2 grupos muestran una evolución negativa en el incremento de la aceleración media y suavidad de movimiento media en el uso del disector, lo que se traduce en un aumento en los cambios bruscos de velocidad y aceleración en sus movimientos. Además, los cirujanos expertos muestran una aceleración y suavidad de movimientos media más elevada en el uso del portaagujas. Tal y como ha ocurrido en el ejercicio de disección, podría ocurrir que la experiencia incite a movimientos más rápidos y, en ocasiones, a movimientos menos delicados.

Esta primera aproximación nos permite contar con un sistema capaz de discernir entre niveles de experiencia. No obstante, si queremos llevar a cabo una evaluación completa de la competencia quirúrgica, aparte de las destrezas quirúrgicas de un cirujano se deben tener en cuenta otros factores como el conocimiento y su disposición frente a posibles complicaciones, entre otros. Esto forma parte de un reto futuro orientado al desarrollo de sistemas avanzados para la evaluación de las competencias quirúrgicas en el que hemos comenzado a trabajar.

En conclusión, este estudio muestra la utilidad del sistema presentado de evaluación objetiva de destrezas psicomotoras laparoscópicas respecto a las 3 tareas analizadas, corte en tejido inorgánico, disección de la serosa gástrica y sutura en tejido orgánico para cirujanos de experiencia intermedia y expertos. El objetivo final perseguido será poder transferir estas habilidades aprendidas y evaluadas al entorno quirúrgico real. Como trabajos futuros se plantea incrementar la muestra objeto de estudio, incluyendo sujetos sin experiencia previa, así como aumentar el número de parámetros a evaluar.

\section{Financiación}

Este trabajo ha sido financiado en parte por la Consejería de Empleo, Empresa e Innovación del Gobierno de Extremadura y el Fondo Social Europeo.

\section{Conflicto de intereses}

Los autores declaran no tener ningún conflicto de intereses.

\section{B I B L I O G R A F Í A}

1. Pérez-Duarte FJ, Sánchez-Margallo FM, Díaz-Güemes I, Sánchez-Hurtado MA, Lucas M, Usón J. Ergonomía en cirugía laparoscópica y su importancia en la formación quirúrgica. Cir Esp. 2012;90:284-91.

2. Figert PL, Park AE, Witzke DB, Schwartz RW. Transfer of training in acquiring laparoscopic skills. Am Coll Surg. 2001;193:533-7.

3. Van Hove PD, Tuijthof GJ, Verdaasdonk EG, Stassen LP, Dankelman J. Objective assessment of technical surgical skills. Br J Surg. 2010;97:972-87.

4. Roberts KE, Bell RL, Duffy AJ. Evolution of surgical skills training. World J Gastroenterol. 2006;12:3219-24.

5. Ritchie WP. Basic certification in surgery by the American Board of Surgery (ABS). What does it mean? Does it have value? Is it relevant? A personal opinion. Ann Surg. 2004;239:133-9.

6. Oropesa I, Sánchez-González P, Lamata P, Chmarra MK, Pagador JB, Sánchez-Margallo JA, et al. Methods and tools for objective assessment of psychomotor skills in laparoscopic surgery. J Surg Res. 2011;171:e81-95.

7. Sánchez-Margallo FM, Asencio JM, Tejonero MC, SánchezHurtado MA, Pérez-Duarte FJ, Usón J, et al. Training design and improvement of technical skills in the transvaginal cholecystectomy (NOTES). Cir Esp. 2009;85:307-13.

8. Chmarra MK, Dankelman J, van den Dobbelsteen JJ, Jansen F-W. Force feedback and basic laparoscopic skills. Surg Endosc. 2008;22:2140-8.

9. Chmarra MK, Grimbergen CA, Jansen FW, Dankelman J. How to objectively classify residents based on their psychomotor laparoscopic skills? Minim Invasive Ther Allied Technol. 2010;19:2-11.

10. Smith CD, Farrell TM, McNatt SS, Metreveli RE. Assessing laparoscopic manipulative skills. Am J Surg. 2001;181:547-50.

11. Rosenbaum DA. Psychological foundations. Human motor control. Academic Press London; 1991. pp 79-118.

12. Sánchez-Margallo JA, Sánchez-Margallo FM, Pagador JB, Gómez EJ, Sánchez-González P, Usón J, et al. Video-based assistance system for training in minimally invasive surgery. Minim Invasive Ther Allied Technol. 2011;20:197-205.

13. Pagador JB, Sánchez-Margallo FM, Sánchez-Peralta LF, Sánchez-Margallo JA, Moyano-Cuevas JL, Enciso-Sanz S, et al. Decomposition and analysis of laparoscopic suturing task using tool-motion analysis (TMA): Improving the objective assessment. Int J Comput Assist Radiol Surg. 2012;7:305-13.

14. Cano AM, Gayá F, Lamata P, Sánchez-González P, Gómez EJ. Laparoscopic tool tracking method for augmented reality surgical applications. Lect Notes Comput Sci. 2008;5104:191-6.

15. Pellen M, Horgan L, Roger Barton J, Attwood S. Laparoscopic surgical skills assessment: Can simulators replace experts? World J Surg. 2009;33:440-7. 
16. Enciso S, Sánchez-Margallo FM, Díaz-Güemes MartínPortugués I, Usón Gargallo J. Validación preliminar del simulador físico Simulap ${ }^{\circledR}$ y de su sistema de evaluación para cirugía laparoscópica. Cir Esp. 2012;90:38-44.

17. Chui CK, Chen G. Correlated system and measurement noise processes. En: Chui CK, Chen G, editores. Kalman filtering: With real-time applications. $4^{\text {th }}$ ed. Berlín: Springer; 2009. p. 49-66.

18. Chmarra MK, Kolkman W, Jansen FW, Grimbergen CA, Dankelman J. The influence of experience and camera holding on laparoscopic instrument movements measured with the TrEndo tracking system. Surg Endosc. 2007;21:2069-75.

19. Chang L, Hogle NJ, Moore BB, Graham MJ, Sinanan MN, Bailey $\mathrm{R}$, et al. Reliable assessment of laparoscopic performance in the operating room using videotape analysis. Surg Innov. 2007;14:122-6.

20. Doyle JD, Webber EM, Sidhu RS. A universal global rating scale for the evaluation of technical skills in the operating room. Am J Surg. 2007;193:551-5.

21. Cleary $\mathrm{K}$, Peters TM. Image-guided interventions: Technology review and clinical applications. Annu Rev Biomed Eng. 2010;12:119-42.

22. Krupa A, Member A, Gangloff J, Doignon C, Mathelin MFD, Morel G, et al. Autonomous 3-D positioning of surgical instruments in robotized laparoscopic surgery using visual servoing. IEEE Trans Rob Autom. 2003;19:842-53.

23. Rosen J, Brown JD, Barreca M, Chang L, Hannaford B, Sinanan M. The BlueDRAGON - a system for monitoring the kinematics and the dynamics of endoscopic tools in minimally invasive surgery for objective laparoscopic skill assessment. Stud Health Technol Inform. 2002;85:412-8.

24. Leong JJ, Nicolaou M, Atallah L, Mylonas GP, Darzi AW, Yang GZ. HMM assessment of quality of movement trajectory in laparoscopic surgery. Med Image Comput Comput Assist Interv. 2006;9:752-9.

25. Clarke JV, Deakin a H, Nicola C, Picard F. Measuring the positional accuracy of computer assisted surgical tracking systems. Comput Aided Surg. 2010;15:13-8.

26. Pellen MGC, Horgan LF, Barton JR, Attwood SE. Construct validity of the ProMIS laparoscopic simulator. Surg Endosc. 2009;23:130-9.

27. Yamaguchi S, Yoshida D, Kenmotsu H, Yasunaga T, Konishi K, Ieiri S, et al. Objective assessment of laparoscopic suturing skills using a motion-tracking system. Surg Endosc. 2011;25:771-5

28. Van Sickle KR, McClusky DA, Gallagher G, Smith CD. Construct validation of the ProMIS simulator using a novel laparoscopic suturing task. Surg Endosc. 2005;19: 1227-31.

29. Moorthy K, Munz Y, Dosis A, Bello F, Chang A, Darzi A. Bimodal assessment of laparoscopic suturing skills: Construct and concurrent validity. Surg Endosc. 2004;18:1608-12.

30. Brydges R, Classen R, Larmer J, Xeroulis G, Dubrowski A. Computer-assisted assessment of one-handed knot tying skills performed within various contexts: A construct validity study. Am J Surg. 2006;192:109-13. 